\title{
Anticancer drug targets: approaching angiogenesis
}

\author{
Eli Keshet ${ }^{1}$ and Shmuel A. Ben-Sasson ${ }^{2}$ \\ ${ }^{1}$ Department of Molecular Biology, and \\ ${ }^{2}$ Department of Experimental Medicine and Cancer Research, The Hebrew University-Hadassah Medical School, \\ Jerusalem 91120, Israel
}

Address correspondence to: Eli Keshet, Department of Molecular Biology, The Hebrew University-Hadassah Medical School, Jerusalem 91120, Israel. E-mail: keshet@cc.huji.ac.il.

For more than 50 years, a direct strategy dominated the field of cancer therapy. The selected target was the tumor cell itself; any cytotoxic drug that could kill tumor cells in vitro was by definition a candidate for in vivo chemotherapy. It soon became apparent, however, that normal cells could also be susceptible to the same spectrum of drugs. Moreover, because of the inherent genetic instability of neoplastic cells, exposure to chemotherapy eventually results in the selection of drug-resistant clones. The indirect strategy of antiangiogenic therapy provides an alternative that uses the evolving vasculature, which nourishes the growing tumor, as the prime target. With this approach, oncologists no longer need to restrict their attention to the individual cancer cell but may focus on its tissue context in general, and on angiogenesis, the process of blood-vessel formation, in particular. This conceptual framework, which has guided the search for new methods to identify novel anticancer drugs, is the subject of this Perspective.

\section{Strategic considerations}

The concept of cancer antiangiogenic therapy stems from Folkman's initial proposition that the expansion of the tumor mass beyond a size of a few cubic millimeters totally depends on de novo formation of a vascular network that provides the growing tumor with oxygen and essential nutrients (1). This thesis, now confirmed by a large body of experimental evidence, implies that tumors can potentially be starved to death by inhibiting their neovascularization. Viewing the tumor as an ecosystem involving reciprocal paracrine interactions between tumor cells and endothelial cells further underscores the importance of a persistent vascular supply for optimal tumor growth (2).

Tumor angiogenesis is generally viewed as a consequence of the activation of an angiogenic switch - a discrete genetic event in the succession of genetic alterations underlying tumor progression that endows the tumor with the ability to recruit blood vessels from the neighboring tissue. In several animal models, a discrete angiogenic switch has been demonstrated during early stages of tumor development that preceded the appearance of large malignant tumors (3).

In principle, targeting the tumor vasculature rather than targeting tumor cells (an approach that is considered by Ohh and colleagues [4] in this issue of the JCI, as well as by the other authors in this Perspectives series) has 2 remarkable advantages. First, because all solid tumors (and probably many leukemias [5]) are angiogensis-dependant, this approach circumvents the need to tailor therapy to the unique genetic makeup of an individual tumor. Second, the targeted vascular endothelial cells are normal, genetically stable cells, and therefore are less likely than are tumor cells to become drug-resistant. Thus, once a genuine antiangiogenic therapy is proven effective in a clinical trial, it could become the major or even the sole anticancer therapy.

How realistic are the prospects of antiangiogenic therapy? The answer to this question depends on the ability to inhibit angiogenesis specifically, without damaging any other tissue. Thus, antiangiogenic therapy must be endothelial cell-specific and must distinguish between tumor and normal vasculatures. Another issue concerns the overall course of antiangiogenic therapy: will it necessarily be chronic in nature, only keeping the tumor from growing bigger, or is it possible that antiangiogenic therapy will also induce tumor regression? Because of our limited understanding of angiogenesis in general and tumor angiogenesis in particular, we are still far from being able to provide a comprehensive answer.

Hopes for inhibiting ongoing tumor neovascularization without inducing adverse effects on the host circulatory system rest in part on the observation that the vasculature in normal adults is generally quiescent, with only $0.01 \%$ of endothelial cells undergoing cell division at any given time. In tumors, the fraction of cycling endothelial cells might be $2-3$ orders of magnitude higher. Therefore, antagonizing endothelial cell proliferation is likely to have a minimal effect on the normal vasculature. Anticipated exceptions are the impairment of reproductive cycles in premenopausal women (due to the fact that hormone-driven angiogenesis naturally takes place in the ovary and endometrium) and a compromised capacity for angiogenesis associated with wound healing. These foreseen complications, however, are considered acceptable in face of the risk of tumor progression.

Another distinguishing characteristic of tumor vessels that might be exploited in selectively targeting them is their relative state of immaturity. Ongoing neovascularization and constant remodeling of tumor vessels (evidenced by regressive changes taking place concomitantly with vessel proliferation) account for the fact that, at any given time during the course of tumor growth, a significant fraction of tumor vessels is poorly structured and immature. One hallmark of the immature vessels present in tumors is incomplete coating with periendothelial 
cells, resulting from the fact that the recruitment of these smooth muscle-like cells lags behind the initial formation of the endothelial plexus. A greater vulnerability of immature vessels, or the differential dependence of immature vessels on specific survival factors might in theory be exploited to enforce vessel regression.

At the molecular level, qualitative differences between tumor vessels and established normal vessels might be reflected in the preferential representation of certain cell-surface proteins on angiogenic tumor vessels. For example, VEGF receptors and $\alpha_{v} \beta_{3}$ and $\alpha_{v} \beta_{5}$ integrins are relatively weakly expressed in normal endothelia, and might provide useful targets for inhibition of tumor vessel angiogenesis.

\section{Tipping the balance}

In general, angiogenesis is controlled by a balance between proangiogenic and antiangiogenic factors. These can be circulatory factors or can act locally as paracrine factors. Obviously, if we know the molecular nature of the proangiogenic signals, we can try to antagonize them, but (perhaps not surprisingly) this fundamental process is regulated by many factors and at multiple levels. Intricate control mechanisms have evolved to accommodate situations requiring a fast transition from long-term quiescence to a swift, localized angiogenic response to meet the emerging needs of the tissue. Angiogenesis is known to be activated by diverse physiologic triggers such as those induced by perturbations in oxygen homeostasis and those regulated by hormonal cues. Yet physiologic angiogenesis (e.g., in the reproductive system) and corrective angiogenesis (e.g., woundhealing angiogenesis and formation of collateral vessels) are self-limiting processes. In the case of spontaneous tumors, a long latent period may precede neovascularization. The stochastic nature of the angiogenic switch implies that mediators that do not play a role in physiologic angiogenesis might also be involved. Both positive regulators (so-called angiogenic factors) and natural inhibitors of angiogenesis exist, and even coexist within the same tissue. The relatively large number of molecules shown to be proangiogenic in a model system is probably partly due to the promiscuity of the model system used. Nevertheless, it also reflects the facts that a number of diffusable proteins with proangiogenic activity exist and that they may cooperate and even have a synergistic effect on the magnitude of the angiogenic response. It is also possible that in different tumor settings, the angiogenic process is driven by varying factors or sets of angiogenic stimuli.

Despite this daunting complexity, the "balance hypothesis" described above depicts the angiogenic switch as the net result of the activity of angiogenic stimulators and inhibitors, suggesting that counteracting even a single major angiogenic factor could shift the balance toward inhibition (3). Therefore, it should not be necessary to define all angiogenic factors that are elaborated by a particular tumor in order to blunt tumor angiogenesis.

Likewise, there is a growing list of allegedly naturally occurring antiangiogenic factors. In this case, the choice is simpler: each of them is in principle a candidate for antiangiogenic therapy. However, only a few of them have passed an in vivo test of tumor growth inhibition in a conventional animal model. Thrombospondin- 1 was the first bona fide antiangiogenic factor to be discovered (6). In a series of publications, Bouck and colleagues showed that the level of production of this naturally occurring glycoprotein is inversely correlated with the level of tissue angiogenesis and tumorigenesis in vivo. Thrombospondin- 1 and -2 remain the most outstanding examples for the mechanism of fine-tuning of angiogenesis by extracellular proteins. Further attempts were made to dissect the molecular structure of this large protein and derive from it peptides with antiangiogenic activity.

Overall, many of the signals controlling the process of angiogenesis are mediated by specific interactions between proteins, including ligand-receptor, cell-extracellular matrix (ECM), and antiangiogenic factor interactions. Thus, the ability to identify peptide domains that are specifically involved in tumor angiogenesis holds great promise. A recently described and powerful methodology - isolation of peptides from phage display libraries that bind to proteins that are preferentially expressed on tumor blood vessels - has proved useful in uncovering receptors that are selectively expressed on tumor vasculature (7). A chimeric peptide containing a tumor blood vessel homing motif and a proapoptotic peptide was selectively toxic to angiogenic endothelial cells and showed anticancer activity in mice (8).

\section{Antagonizing proangiogenic activities}

The angiogenic response that follows the reception and transduction of the angiogenic stimulus comprises a complex series of events. This includes local degradation of the basement membrane, directional migration of the underlying endothelial cells, invasion of the surrounding stroma, endothelial cell proliferation, capillary tube morphogenesis, coalescence of capillaries into larger vessels, vascular pruning, and acquisition of a periendothelial cell coating. In essence, tumor neovascularization could be inhibited at each of these strategic junctions. An important consideration is whether all proangiogenic stimuli converge on a common pathway or different angiogenic factors activate distinct angiogenic pathways. Evidence for the latter was provided by showing that VEGF-induced angiogenesis and bFGF-induced angiogenesis in model systems involve different angiogenesis-specific integrin receptors (9). However, the relevance of this finding to natural tumor angiogenesis is unknown. In practice, we can discover and design a very useful antiangiogenic therapy even without having a full knowledge of the entire angiogenic process. Yet several neglected aspects of angiogenesis, such as the mechanism of natural angiogenesis inhibitors and the process of tube morphogenesis, deserve more intensive exploration.

A comprehensive account of all candidate proangiogenic and antiangiogenic molecules that are currently under study is beyond the scope of this article, but we describe below a selection of candidate drugs that have been found to be angiogenesis-specific and effective as anticancer agents in vivo, and that are either in clinical trial or on the verge of being so.

With regard to the preferred tumor-angiogenic factor to be antagonized, extensive research from many laboratories has generated an exhaustive catalogue of angio- 
genic factors produced by a wide range of naturally occurring tumors. Out of these studies, VEGF has emerged as the single most commonly upregulated angiogenic factor in both grafted and naturally occurring tumors. In conjunction with the well established relevance of VEGF to embryonic and physiologic angiogenesis, this consideration renders VEGF a prime target for antiangiogenic therapy.

VEGF, more recently dubbed VEGF-A, is a prototypic protein in a growing family of secreted ligands. Additional family members have proangiogenic activity, and one member (VEGF-C) is also distinguished by lymphangiogenic activity. Alternative splicing modes of VEGF account for the generation of several isoforms; some are mostly sequestered pericellularly and others are freely diffusable. VEGF exerts its biological activity by binding to its tyrosine kinase receptors, VEGF-R1 (Flt-1) and VEGF-R2 (Flk$1 / \mathrm{KDR}$ ), and to the auxiliary receptor neuropilin.

VEGF plays essential roles in both vasculogenesis and angiogenesis, and is considered to be an embryonic cardiovascular morphogen. VEGF is tightly regulated during development, as demonstrated by the embryonic lethality of heterozygous mice carrying only one wildtype Vegf allele. In tumors, the escape of VEGF from its otherwise tight control is in some cases achieved through activating mutations in oncogenes such as ras. In other cases, misregulation is a consequence of a lossof-function mutation in tumor suppressors such as VHL. A gross correlation between the level of VEGF expression and tumor vascularity has been noted, and has been further substantiated through the use of experimental tumor systems engineered to overexpress VEGF. Interestingly, experimental overexpression of VEGF results in abnormally large, hyperfused vessels that are reminiscent of vessels found in hemangioblastoma tumors that naturally express exceedingly high levels of VEGF.

Preclinical studies have used different classes of VEGF antagonists, including anti-VEGF neutralizing antibodies, soluble versions of VEGF-R1 and -R2, and inhibitors of the VEGF-R2 tyrosine kinase. Tumor-bearing nude mice were used to evaluate the inhibitory effect of these drugs on tumor growth and neovascularization. Encouraging positive results, manifested by significant inhibition of tumor growth and evidence for reduced vasculature $(10,11)$, led to the advancement of 4 drugs into clinical trials: 3 drugs that block VEGF receptor signaling (currently in phase I/II) and a humanized VEGF-neutralizing $\mathrm{mAb}$ (currently in phase II).

While awaiting the results of the clinical trials, some issues pertinent to anti-VEGF therapy should be considered. First, the major physiologic role of VEGF in the adult is to promote corrective angiogenesis in response to perturbation of oxygen homeostasis. Accordingly, VEGF is strongly induced once hypoxia or hypoglycemia are sensed by oxygen-deprived cells. Tumors generally maintain the capacity to upregulate VEGF in response to hypoxia, suggesting that irrespective of the nature of a genetic angiogenic switch, overall tumor vascularity might be increased by hypoxia-induced VEGF. In fact, tumors that have been compromised in their ability to mount a hypoxic response (e.g., tumor cells carrying a null mutation in the transcription factor HIF-1) are sig- nificantly impaired in their vascular function (12). Therefore, whatever antiangiogenic method is used, vascular collapse is expected to result in extensive hypoxia, which in turn will elicit a second angiogenic wave mediated by VEGF. Antagonizing VEGF might also eliminate of an anticipated second angiogenic wave.

Second, VEGF also functions as a survival factor for immature blood vessels, which become VEGF independent only upon their maturation and engagement with periendothelial cells. Consequently, a newly formed vascular plexus will regress if VEGF is prematurely withdrawn. In fact, this is a natural mechanism to fine-tune vascular density through eliminating surplus vessels. The immature vessels frequently found in tumors may therefore represent a subset of vulnerable vessels requiring the continuous presence of VEGF. Recent studies have indeed demonstrated that regression of immature vessels can be induced by VEGF deprivation. Furthermore, it has been argued that early regression of vessels as a consequence of androgen-ablation therapy in prostate carcinoma is due to suppression of androgen-regulated VEGF production (13). The dual action of VEGF as an angiogenic factor and a vascular survival factor may provide a mechanistic explanation to the thesis that VEGF deprivation may lead not only to inhibition of further angiogenesis, but also to regression of preformed tumor vessels. One point of concern is the fact that VEGF also plays a role in normal vessel functioning, primarily in controlling vessel permeability. It remains to be ascertained whether systemic administration of VEGF-blocking reagents will not adversely affect normal vascular functions.

\section{Harnessing natural antiangiogenic factors}

In pioneering work, Folkman and O'Reilly took advantage of the biological phenomenon that in some experimental models, a primary tumor can suppress lung metastases or the growth of another tumor at a remote site. Folkman conjectured that this inhibitory effect might be due to the production of a circulating antiangiogenic factor by the primary tumor. Thus, in the immediate vicinity of the primary tumor, proangiogenic signals override the putative antiangiogenic signals, resulting in continuous tumor growth. However, at remote sites the balance tilts in the other direction because proangiogenic factors have a relatively short half-life, whereas antiangiogenic factors might last in the circulation much longer, creating a net inhibitory effect. Based on this working hypothesis, O'Reilly et al. chose a mouse tumor model that strongly suppresses lung metastases. From the urine of these tumor-bearing mice, O'Reilly and colleagues purified a protein that specifically inhibited endothelial cell proliferation in vitro without affecting other cell types (14). Sequence analysis revealed that the isolated protein is a $38-\mathrm{kDa}$ internal fragment of the serum protein plasminogen, which itself lacks any antiangiogenic activity. This novel peptide, named angiostatin, can be a cleavage product of macrophage elastase or can be produced by the action of other proteolytic enzymes.

A second antiangiogenic peptide was isolated by the same group, using a similar procedure, and was named endostatin. Endostatin is a $20-\mathrm{kDa} \mathrm{COOH}$-terminal 
fragment of collagen XVIII, a component of the bloodvessel wall that participates in pattern formation during embryonic development. Endostatin was shown to inhibit VEGF-induced endothelial cell migration in vitro and to have antitumor activity in vivo, without any apparent signs of toxicity (15).

Each of these angiogenesis inhibitors almost completely suppresses the growth of a variety of tumors in mice, including xenografts of human tumors of different origins, with endostatin being more potent $(15,16)$. When given in combination, angiostatin and endostatin act synergistically and cause a partial regression of the tumor burden in a transgenic mouse model of tumor progression (17). To demonstrate that antiangiogenic therapy is refractory to the development of drug resistance, endostatin treatment of tumor-bearing mice was cycled, mimicking cancer recurrence after tumor relapse. As expected, the efficacy of the antiangiogenic treatment did not fade with time. Surprisingly, however, after several cycles of treatment the tumor did not grow back, even though endostatin injection was discontinued (18). Although different groups used different constructs of endostatin, it is noteworthy that the most dramatic effects of endostatin were achieved with a non-refolded, precipitated form of the peptide, produced in bacteria (15).

The way in which angiostatin and endostatin were isolated suggests that other proteins with antiangiogenic activity have yet to be discovered. Although we have only a limited sample, some common features emerge. First, we are dealing with completely nontoxic, nonimmunogenic, natural body products that are specific antiangiogenic agents. Second, both inhibitors are liberated by proteolysis from precursor proteins, which in their intact form play other roles. Interestingly, a similar principle is shared with other biological systems such as the complement activation cascade. Unfortunately, however, almost nothing is known about the mechanism of action of natural angiogenesis inhibitors.

Practical considerations regarding the required therapeutic effective dose (in the range of $10-100 \mathrm{mg}$ per $\mathrm{kg}$ body weight per day in mice) and the need for continuous injections provide a strong incentive to develop innovative methods to produce these proteins in vivo. Introducing DNA that codes for an antiangiogenic factor is one way to circumvent the need for daily injection. An intriguing strategy is that of inducing the release of antiangiogenic peptides from their abundant endogenous precursor proteins. The foundations for this novel approach were laid by Gately et al. (19), who delineated the conditions for the conversion of plasminogen to angiostatin. Plasminogen activators, such as tissue-type plasminogen activator or streptokinase, are routinely used in the clinic. In combination with a sulfhydryl donor such as L-cysteine or captopril, these enzymes generate angiostatin from plasminogen. The processed protein is active in vivo, and was shown to suppress lung metastases in a tumor model (19). These findings can be translated into human therapy with relative safety, thanks to long experience with these drugs. Beyond its expected immediate benefit, this work encourages a search for other proteolytic enzymes and accessory molecules that might contribute to the formation of addi- tional antiangiogenic peptides. In other words, this biochemical scheme provides an algorithm that can speed up the discovery of other novel peptides. The rapid development of analytical tools in the field of proteomics makes such a mass screening feasible.

\section{Modulation of endothelial cell-ECM interactions}

Initially viewed as merely a physical barrier, the ECM is now recognized as having a profound effect on the angiogenic phenotype through multiple dynamic interactions with endothelial cells and the transduction of signals by cross-linking integrin receptors on endothelial cells. These adhesive interactions provide potential means for intervention aimed to inhibit tumor neovascularization. Parallels between the ECM degradation required for endothelial cell invasion and that required for the spreading of metastatic tumor cells have been drawn, engendering strategies for controlling the process and applying antiproteolytic techniques (20). In addition, some aspects of matrix metalloproteinase (MMP) action appear to be specific for angiogenesis. For example, certain angiogenic factors are sequestered in the ECM in an inactive form and require MMP-mediated proteolysis to be activated and released from their storage depots (21). Ongoing antiangiogenic therapeutic approaches to target MMP activity use general class inhibitors that are selective, but not specific, for some MMP family members. This topic was recently reviewed in a Perspective article in the JCI that concluded that much work is needed for a better understanding of the specific MMPs to be targeted and their precise role in the angiogenic response in order to develop more specific inhibitors with fewer side effects (22).

Another potential point of intervention is at the level of integrin-mediated adhesion of endothelial cells to ECM components. Specifically, antagonists of $\alpha_{v}$ integrins have been shown to disrupt tumor angiogenesis in vivo, and are currently in phase II clinical trials. The rationale for this approach is based on findings from Elicieri and coworkers that $\alpha_{v} \beta_{3}$ integrin receptors are present on the surface of angiogenic endothelium but not on quiescent endothelium. Furthermore, ligation of $\alpha_{v} \beta_{3}$ receptors promotes the survival of endothelial cells engaged in ongoing angiogenesis, as evidenced by the fact that $\alpha_{v} \beta_{3}$ antagonists administered during angiogenesis induce endothelial cell apoptosis. Positive preclinical inhibition studies using human tumor xenografts and tumors in patches of human skin in SCID chimeric mice have prompted clinical trials using a humanized form of the $\alpha_{v} \beta_{3}$ mAb LM609 (Vitaxin). For a recent review on their mechanisms of action and clinical development targeting the $\alpha_{v}$ integrins, see ref. 23 .

\section{Epilogue}

The most important achievement of tumor angiogenesis research to date is the proof of the principle that antiangiogenic therapy is a viable option for the treatment of cancer. These prospects have fueled intensive research by scientists in both academia and the pharmaceutical industry. We are dealing with an unfolding chapter in developmental biology, with many questions still open regarding the nature of control mechanisms at the cellu- 
lar, tissue, and organismal levels. In some cases, we are still missing a conceptual framework; in others, we are in the process of filling in the details. For example, the prevailing view of an avascular tumor mass acquiring the ability to recruit blood vessels from the neighboring tissue needs to be modified somewhat in order to accommodate recent observations. First, a subset of tumors and metastases initially grows by coopting existing host vessels before the induction of tumor neovascularization (24). Second, certain tumors (e.g., uveal melanomas) may generate vascular channels lacking endothelial cell lining (25). Nonetheless, most tumors are angiogenesis dependent at crucial stages of their growth.

In addition to the therapeutic targets described above, interventions at other stages of the angiogenic process are being explored. The realizations that vessel disassembly is required for the initiation of angiogenesis, and that the recruitment of pericytes is a key step in vessel maturation underscores the pivotal role of factors governing endothelial-periendothelial cell associations. In this respect, the roles of angiopoietin-1 in pericyte recruitment and vessel tightening, and the opposing effect of angiopoietin-2 in vessel destabilization (26) can be exploited to modify the phenotypes of tumor vessels. If some angiogenesis-specific master transcription factor can be identified upon which different angiogenic stimuli converge, such a factor would be a prime target for antiangiogenic therapy. Since cell-cell adhesion within the endothelium is mediated by VE-cadherin, perhaps this molecule can be targeted selectively in tumors. Other factors that regulate cell-ECM interactions, such as the long-sought and recently characterized heparanase, could also be important as prospective targets (27).

The identification of receptors for the newly discovered antiangiogenic proteins might open the way for the discovery of small molecules with therapeutic value, either through a rational drug design or by a brute-force, high-throughput screening. Still, a word of caution is appropriate: obtaining a low-molecularweight compound with exclusive specificity for endothelial cells remains a great challenge. For example, although the ATP-binding motif of the VEGF receptor's kinase domain might seem an attractive target for small-molecule therapy, each of the estimated 2,000 different protein kinases in the human body binds ATP by a similar structural module. Similar concerns about nonspecific cross-toxicity apply to other possible classes of target proteins.

\section{Acknowledgments}

Editorial constraints prevent the referencing of some seminal studies. For further information about the entire spectrum of angiogenesis inhibitors currently in clinical trials, the reader is referred to the NCI web site: http://cancertrials.nci.nih.gov.
1. Folkman, J. 1971.Tumor angiogenesis: therapeutic implications. N. Engl. J. Med. 285:1182-1186.

2. Rak, J., Filmus, J., and Kerbel, R.S. 1996. Reciprocal paracrine interactions between tumour cells and endothelial cells: the 'angiogenesis progression' hypothesis. Eur. J. Cancer. 32A:2438-2450.

3. Hanahan, D., and Folkman, J. 1996. Patterns and emerging mechanisms of the angiogenic switch during tumorigenesis. Cell. 86:353-364.

4. Ohh, M., et. al. 1999. Synthetic peptides define critical contacts between elongin C, elongin B, and the von Hippel-Lindau protein. J. Clin. Invest. 104:1583-1591

5. Perez-Atayde, A.R., et al. 1997. Spectrum of tumor angiogenesis in the bone marrow of children with acute lymphoblastic leukemia. Am. J. Pathol. 150:815-821.

6. Good, D.J., et al. 1990. A tumor suppressor-dependent inhibitor of angiogenesis is immunologically and functionally indistinguishable from a fragment of thrombospondin. Proc. Natl. Acad. Sci. USA. 87:6624-6628.

7. Pasqualini, R., and Ruoslahti, E. 1996. Organ targeting in vivo using phage display peptide libraries. Nature. 380:364-366.

8. Ellerby, H.M., et al. 1999. Anti-cancer activity of targeted proapoptotic peptides. Nat. Med. 5:1032-1038.

9. Friedlander, M., et al. 1995. Definition of two angiogenic pathways by distinct $\alpha_{v}$ integrins. Science. 270:1500-1502.

10. Kim, K.J., et al. 1993. Inhibition of vascular endothelial growth factorinduced angiogenesis suppresses tumour growth in vivo. Nature. 362:841-844.

11. Fong, T.A., et al. 1999. SU5416 is a potent and selective inhibitor of the vascular endothelial growth factor receptor (Flk-1/KDR) that inhibits tyrosine kinase catalysis, tumor vascularization, and growth of multiple tumor types. Cancer Res. 59:99-106.

12. Carmeliet, P., et al. 1998. Role of HIF- $1 \alpha$ in hypoxia-mediated apoptosis, cell proliferation and tumor angiogenesis. Nature. 394:485-490.

13. Benjamin, L., Golijanin, D., Itin, A., Pode, D., and Keshet, E. 1999. Selective ablation of immature blood vessels in established human tumors follows vascular endothelial growth factor withdrawal. J. Clin. Invest. 103:159-165.

14. O'Reilly, M.S., et al. 1994. Angiostatin: a novel angiogenesis inhibitor that mediates the suppression of metastases by a Lewis lung carcinoma. Cell. 79:315-328.

15. O'Reilly, M.S., et al. 1997. Endostatin: an endogenous inhibitor of angiogenesis and tumor growth. Cell. 88:277-285.

16. O'Reilly, M.S., Holmgren, L., Chen, C., and Folkman, J. 1996. Angiostatin induces and sustains dormancy of human primary tumors in mice. Nat. Med. 2:689-692.

17. Bergers, G., Javaherian, K., Lo, K.M., Folkman, J., and Hanahan, D. 1999. Effects of angiogenesis inhibitors on multistage carcinogenesis in mice. Science. 284:808-812.

18. Boehm, T., Folkman, J., Browder, T., and O'Reilly, M.S. 1997. Antiangiogenic therapy of experimental cancer does not induce acquired drug resistance. Nature. 390:404-407.

19. Gately, S., et al. 1997. The mechanism of cancer-mediated conversion of plasminogen to the angiogenesis inhibitor angiostatin. Proc. Natl. Acad. Sci. USA. 94:10868-10872.

20. Liotta, L.A., Steeg, P.S., and Stetler-Stevenson, W.G. 1991. Cancer metastasis and angiogenesis: an imbalance of positive and negative regulation. Cell. 64:327-336.

21. Vu, T.H., et al. 1998. MMp-9/gelatinase B is a key regulator of growth plate angiogenesis and apoptosis of hypertrophic chondrocytes. Cell. 93:411-422.

22. Stetler-Stevenson, W.G., 1999. Matrix metalloproteinases in angiogenesis: a moving target for therapeutic intervention. J. Clin. Invest. 103:1237-1241.

23. Elicieri, B.P., and Cheresh, D.A. 1999. The role of $\alpha_{v}$ integrins during angiogenesis: insights into potential mechanisms of action and clinical development. J. Clin. Invest. 103:1227-1230.

24. Holash, J., et al. 1999. Vessel cooption, regression, and growth in tumors mediated by angiopoietins and VEGF. Science. 284:1994-1998.

25. Maniotis, A.J., et al. 1999. Vascular channel formation by human melanoma cells in vivo and in vitro: vasculogenic mimicry. Am. J. Pathol. 155:739-752.

26. Gale, N.W., and Yancopoulos, G.D. 1999. Growth factors acting via endothelial cell-specific receptor tyrosine kinases: VEGFs, angiopoietins, and ephrins in vascular development. Genes Dev. 13:1055-1066.

27. Vlodavsky, I., et al. 1999. Mammalian heparanase: gene cloning, expression and function in tumor progression and metastasis. Nat. Med. 5:793-802. 